Cahiers d'études africaines

$179-180 \mid 2005$

Esclavage moderne ou modernité de l'esclavage?

\title{
Inégalité, exclusion, représentations sur les Hautes Terres centrales de Madagascar
}

L. N. Razafindralambo

\section{OpenEdition}

1 Journals

Édition électronique

URL : http://journals.openedition.org/etudesafricaines/15038

DOI : 10.4000/etudesafricaines. 15038

ISSN : 1777-5353

Éditeur

Éditions de l'EHESS

\section{Édition imprimée}

Date de publication : 19 décembre 2005

Pagination : 879-904

ISBN : 978-2-7132-2049-4

ISSN : 0008-0055

Référence électronique

L. N. Razafindralambo, «Inégalité, exclusion, représentations sur les Hautes Terres centrales de Madagascar », Cahiers d'études africaines [En ligne], 179-180 | 2005, mis en ligne le 01 janvier 2007, consulté le 17 juin 2020. URL : http://journals.openedition.org/etudesafricaines/15038; DOI : https:// doi.org/10.4000/etudesafricaines.15038 


\title{
CA I R N
}

chercher : repérer : avancer

Cet article est disponible en ligne à l'adresse :

http://www.cairn.info/article.php?ID_REVUE=CEA\&ID_NUMPUBLIE=CEA 179\&ID_ARTICLE=CEA 1790879

Inégalité, exclusion, représentations sur les Hautes Terres centrales de Madagascar

\author{
par L. N. RAZAFINDRALAMBO
}

\section{Editions de l'EHESS | Cahiers d'études africaines}

2005/3-4 - 179

ISSN 0008-0055 | ISBN 2713220491| pages 879 à 904

Pour citer cet article :

- Razafindralambo L., Inégalité, exclusion, représentations sur les Hautes Terres centrales de Madagascar, Cahiers d'études africaines 2005/3-4, 179, p. 879-904.

Distribution électronique Cairn pour Editions del'EHESS.

(C) Editions de l’EHESS. Tous droits réservés pour tous pays.

La reproduction ou représentation de cet article, notamment par photocopie, n'est autorisée que dans les limites des conditions générales d'utilisation du site ou, le cas échéant, des conditions générales de la licence souscrite par votre établissement. Toute autre reproduction ou représentation, en tout ou partie, sous quelque forme et de quelque manière que ce soit, est interdite sauf accord préalable et écrit de l'éditeur, en dehors des cas prévus par la législation en vigueur en France. Il est précisé que son stockage dans une base de données est également interdit. 


\section{N. Razafindralambo}

\section{Inégalité, exclusion, représentations sur les Hautes Terres centrales de Madagascar*}

Tous les habitants de Madagascar sont proclamés libres. Ainsi l'a stipulé le premier article de l'arrêté du 26 septembre 1896. La conséquence de cet arrêté, ainsi que de celui qui a été proclamé le 28 février 1897 abolissant la royauté, aurait dû être la suppression des quatre principales catégories hiérarchiques subdivisant la société de $\mathrm{l}^{\prime} I m e r i n \mathrm{a}^{1} \mathrm{du} \mathrm{XIX}^{\mathrm{e}}$ siècle : andriana, hova, mainty et andevo, notamment parce que l'arrêté de 1896 a libéré les andevo de leurs anciens maîtres².

Privilèges accordés à certains andriana, comme le port de l'ombrelle rouge, la consommation de l'arrière-train des zébus tués, l'ordre de préséance dans les discours ou lors de la remise de la pièce non coupée $h a s i n a^{3}$, les formules de salutation, etc. matérialisent la hiérarchie.

L'esclavage a-t-il existé à Madagascar ? Cette question soulève le problème de l'application du même terme à différents systèmes qui ont existé à différentes époques. Domenichini-Ramiaramanana et Domenichini (1997, 1998) affirment que les andevo ne sont pas des esclaves ; seuls les Mozambiques, originaires d'Afrique de l'Est, l'ont été. Ils rejoignent sur ce point C. Meillassoux (1986: 100) qui définit l'esclave comme un captif arraché à sa société d'origine et introduit comme étranger dans la société esclavagiste. Ce critère n'est pas retenu par Patterson (1982) pour qui l'esclave est un mort social. Il ne peut avoir de filiation ou d'autres liens de parenté.

* Je tiens à remercier Sophie Blanchy d'avoir accepté de lire cet article.

1. Région des Hautes Terres centrales, dans laquelle se trouve la capitale actuelle de Madagascar.

2. Les andevo eux-mêmes pouvaient être maîtres d'andevo.

3. Le hasina est une notion fondamentale des sociétés malgaches, et contient plusieurs significations : pièce de monnaie présentée comme hommage au souverain lors de différentes circonstances (les subdivisions de la pièce servent aux transactions commerciales); "vertu, force, puissance, grâce, propriété naturelle ou surnaturelle, efficacité, sainteté, vertu attachée à un objet, ce qui rend digne de respect » (ABINAL \& MALZAC 1993) ; et « sel, saveur, goût» (ibid.). Le hasina est également une composante de la personne. 
Cette «incapacité sociale de l'esclave à se reproduire socialement, c'està-dire l'incapacité juridique d'être "parent" » constitue le trait qui apparaît dans toutes les formes d'esclavage (Meillassoux 1986: 35).

Certains anciens andevo sont rentrés dans leur région d'origine. On dispose de peu d'informations relatives à la manière dont ils se sont réintégrés à leur ancien groupe (Rantoandro 1997: 279-280). D'autres sont partis s'établir sur de nouvelles terres, mais ils ne semblent pas avoir laissé derrière eux les «stigmates » de leur ancien statut, alors qu'ils sont des nouveaux venus dans ces régions (Evers). Beaucoup sont cependant restés dans les villages de leurs anciens maîtres.

Plus d'un siècle après cette libération, les termes désignant les quatre catégories sont toujours utilisés. On continue à appeler andriana, hova ou mainty ceux qui sont considérés respectivement comme descendants d'anciens andriana, d'anciens hova ou d'anciens mainty. De même que ceux qui sont considérés comme descendants des anciens andevo libérés en 1896 sont toujours appelés andevo. D'autre part, les olompotsy (personne blanche) désignaient autrefois toute la population libre (andriana, hova et mainty) ${ }^{4}$. Désormais, les descendants des anciens andriana et hova sont les seuls fotsy (blanc) ou olompotsy, tandis que les supposés descendants des anciens mainty et andevo sont regroupés sous le terme mainty (noir) ou olomainty (personne noire).

L'ancien système hiérarchique perdure ainsi, bien que la libération des anciens andevo ait redéfini les relations entre ceux-ci et les anciens maîtres, et entre leurs descendants. La subsistance des andevo a été assurée par leurs anciens maîtres. Un des éléments qui définissent l'état d'andevo a été leur incapacité à devenir propriétaire ${ }^{5}$, et le fait de quitter la maisonnée des maîtres a laissé la plupart sans ressources. Certains andevo possédaient des biens importants et l'on peut supposer qu'ils ont été parmi les premiers à quitter le village de leur maître.

Les terres ont donc appartenu aux fotsy et leur accès a été limité pour les descendants des anciens andevo. Dans les régions rurales où les habitants vivent principalement de l'agriculture, les mainty doivent travailler sur des terres qui ne leur appartiennent pas, principalement en métayage. Le salariat agricole n'est pas très étendu, notamment parce que les propriétaires fotsy n'ont pas les moyens d'y avoir recours. Les mainty doivent donc dépendre des fotsy pour leur subsistance. Ils constituent une réserve de main-d'œuvre dont ces derniers ont besoin, non seulement pour cultiver leurs terres mais

4. Plusieurs expressions désignent l'affranchissement : nalefa olompotsy (est devenu olompotsy), nofotsina (a été blanchi)... et cela même si l'affranchi intègre un groupe mainty.

5. L'état d'andevo est distinct du statut de libre défini comme les «prérogatives sociales acquises à un individu par la naissance (ou son équivalent, l'adoption) et perdues par la mort (ou son équivalent, la capture ou la déchéance) » (MeILLASSOUX $1975: 21$, n. 16). 
également pour accomplir certaines tâches comme creuser les tombeaux pour les enterrements.

Ces relations d'ordre économique ont été présentées comme la cause du maintien de l'inégalité entre les différentes catégories de la population, entre les descendants des anciens libres (les fotsy) et ceux des anciens andevo (les mainty).

Dans les régions du sud des Hautes Terres, dont nous parlerons dans ce texte, la proximité géographique avec la capitale permet d'accéder à différentes opportunités d'emploi. De plus en plus de personnes travaillent en ville, notamment dans les usines de textile en périphérie de la capitale. Parallèlement, les habitants continuent de pratiquer l'agriculture. Beaucoup de fotsy ont vendu leurs biens immobiliers en quittant leurs villages. Les mainty ont ainsi acquis des terres. Ils continuent cependant à faire du métayage sur les terres des fotsy, ou les louent. Le métayage et la location constituent désormais un apport d'argent et de produits agricoles sur lequel comptent les ménages fotsy. D'autre part, ceux-ci présentent leur besoin de main-d'œuvre comme une dépendance vis-à-vis des mainty qu'il faut dès lors ménager. Et l'on peut constater que de plus en plus de terres restent incultivées. Les relations économiques entre mainty et fotsy sont donc caractérisées par une dépendance réciproque. Cependant, l'inégalité entre eux est maintenue, et ses différentes manifestations dans la vie quotidienne seront présentées dans cet article. Les faits observés à l'époque actuelle seront mis constamment en perspective avec la situation prévalant pendant l'ancien régime royal. L'histoire sert de justification aux différents statuts dont on se réclame : fotsy, andriana ou hova. Le rang des anciens groupes sociaux a été déterminé par leur place dans la formation des différents royaumes, et par leur lien de parenté avec les souverains. À chaque groupe social ont correspondu un statut et un territoire. Lorsqu'un individu déclare qu'il est originaire d'un endroit, cela signifie qu'il revendique le statut du groupe qui y est attaché.

\section{Expression de l'inégalité}

\section{Libres et non-libres : la hiérarchie fondamentale}

L'appartenance aux différentes catégories sociales est déterminée par la naissance. Mais un individu ou un groupe peut être placé dans une catégorie autre que celle à laquelle il a appartenu auparavant; cela relève des prérogatives du souverain et ne concerne cependant que les trois premières catégories, andriana, hova et mainty, qui regroupent les sujets du souverain d'Imerina. La vente transforme en andevo les anciens sujets condamnés pour avoir commis des crimes ou des infractions graves, ou qui n'ont pas 
acquitté leurs dettes ${ }^{6}$, les captifs originaires d'autres régions de Madagascar et ceux amenés d'Afrique de l'Est par la traite. La ligne de démarcation la plus importante est donc celle qui sépare la population libre, les sujets, des andevo: les sujets appartiennent aux fanjakana du souverain, les andevo au fanjakana de leur maître (Domenichini-Ramiaramanana \& Domenichini 1982 : 57-59), le fanjakana pouvant ainsi être défini comme le domaine soumis à une autorité ${ }^{7}$. De ce fait, seul le consentement du maître permet à un andevo de rejoindre la population libre ${ }^{8}$.

Ce consentement n'est cependant pas suffisant pour que l'andevo intègre, ou réintègre, la population libre. Il doit également être accepté par un groupe auquel il offre un zébu qui est consommé lors d'un repas communiel. D'autre part, il présente la pièce non coupée hasina aux autorités qui représentent le souverain pour marquer son nouveau statut de sujet, et donc son changement de fanjakana. L'affranchissement comporte deux points importants : intégration de l'ancien andevo parmi les sujets du souverain, impliquant différents droits mais également des obligations comme la corvée ou le service armé ; intégration dans un groupe, c'est-à-dire que le nouveau libre a désormais le droit d'être enterré dans le tombeau du groupe et d'y prendre une épouse. L'affranchissement accorde ainsi à l'andevo le droit d'établir des relations de parenté, ce que ne permet pas l'état d'andevo: l'andevo n'a pas de parent, n'a pas d'époux reconnu et n'a pas d'enfant, il n'a pas d'ancêtre et ne peut le devenir.

\section{Après 1896}

Par le regroupement des quatre anciennes catégories en deux principales, fotsy et mainty, la ligne séparant la population libre (y compris les mainty) et les andevo s'est ainsi déplacée. L'opposition la plus opérante est désormais entre fotsy et mainty.

Ce déplacement semble être l'aboutissement d'un processus d'amalgame qui a commencé à l'époque royale. Lors de la réorganisation des groupes sociaux à la fin du XVIII ${ }^{\mathrm{e}}$ siècle, les groupes mainty ont été regroupés sous une nouvelle catégorie, les mainty enin-dreny. Comme conséquence de ce regroupement, ils perdent leur fonction guerrière et quittent leur statut privilégié de proches du souverain, à l'exception tout d'abord des Tsiarondahy, troisième groupe mainty, qui restent en permanence sous les armes et

6. Certains condamnés sont punis à devenir andevo à titre définitif, c'est-à-dire sans possibilité d'affranchissement. Pour ceux qui ont commis des crimes moins graves, leur valeur marchande est estimée, et si leur famille peut s'en acquitter, ils retournent à leur ancienne appartenance.

7. Comprenant aussi bien les biens (terres, zébus ou andevo) que l'épouse, les enfants et les autres dépendants.

8. Le maître peut offrir l'affranchissement à l'andevo, ou celui-ci peut se racheter ou être racheté par sa famille. 
demeurent dans l'entourage du souverain, avant d'en être éliminés au $\mathrm{XIX}^{\mathrm{e}}$ siècle. Ce rabaissement des mainty se fait au profit de groupes hova, sur lesquels les mainty ont semblé autrefois avoir la préséance (DomenichiniRamiaramanana \& Domenichini 1980 : 86-87) ${ }^{9}$.

D'autre part, les prisonniers de guerre, constituant la part du souverain dans le partage des prises de guerre, ainsi que certains condamnés, ont été agrégés aux mainty attachés au service du souverain, et plus particulièrement aux Tsiarondahy ${ }^{10}$. Il en a été de même des affranchis, anciens andriana ou hova qui n'ont pas été acceptés par leur groupe d'origine, ou ni anciens andriana ni anciens hova et qui ne peuvent donc s'intégrer à d'autres groupes, comme les Mozambiques affranchis en 1877 par décret royal. Ils ont formé un groupe probablement à part parmi les mainty, le groupe des Zazamanga (enfant bleu) ${ }^{11}$.

Un autre terme, toujours en usage, est ankizy (enfant). Le même terme désigne donc les enfants et les andevo, et souligne de ce fait le statut d'enfant mineur de l'andevo ${ }^{12}$. D'autre part, ankizy rappelle que l'andevo appartient au fanjakana d'un maître au même titre que l'épouse et les enfants de ce dernier. Par contre, l'ankizin-drazana (enfant des ancêtres), toujours transmis par héritage et que le maître n'a pas le droit de vendre, a quasiment fait partie de la famille de ce dernier. Ce terme traduit donc une relation de confiance proche de la relation de parenté entre l'andevo et la famille de son maître.

Les mainty des milieux urbains ont « inventé » d'autres termes. L'utilisation de certains mots permet d'éviter le terme andevo. Comme Baomanga, prénom féminin qui désignait au début la beauté féminine, mainty (Randriamaro 1997b : $367)^{13}$, dimbaka et makossa, font référence au musicien Manu Dibango et au style de musique africain, et les mots tirés des noms de différents groupements réunissant les jeunes des milieux défavorisés (jomaka de ZOAM, dans les années 1970, TTs dans les années 1980) (Ramamonjisoa 1984 : 43). Les fotsy sont appelés tsalo (ibid.). On peut également entendre l'expression madio lamba (litt., ceux qui portent des tissus propres).

9. Le nombre d'officiers supérieurs mainty est passé de 36 avant 1828, 71 avant 1861, à 9 en 1896 (Domenichini-Ramiaramanana \& Domenichini 1980 : 90). Parallèlement, les hova prennent le pouvoir effectif : ils fournissent les principaux membres du gouvernement dont les Premiers ministres, désormais seuls faiseurs de roi.

10. Ceux-ci ont donc appartenu à la population libre, mais d'une part, leur statut est inférieur à leur statut antérieur, et d'autre part, ils ne peuvent plus retourner à leur groupe d'origine, puisque n'étant pas à proprement parler des andevo, ils ne peuvent pas être affranchis.

11. Ce terme continue également d'être utilisé, mais par les descendants mêmes des anciens Mozambiques affranchis (RAKotomalala \& RaZAFIMbelo 1985 : 106).

12. "Ankizy» viendrait d'un terme swahili qui désigne la femme esclave (I. Rakoto, communication personnelle).

13. Baomanga: Bao, prénom féminin, et manga: bleu. La couleur bleue pourrait être le côté positif de la couleur noire (voir également zazamanga). 
Certains mainty âgés choisissent le terme mpanompo (serviteur) pour parler de leurs ancêtres andevo. Le substantif fanompoana désigne aussi bien la servitude ou l'assujettissement que la corvée due par les hommes libres au souverain. Les chrétiens se disent également mpanompon'Andriamanitra (serviteurs de Dieu). Le choix de ce terme atténue ainsi, d'une certaine manière, le «statut servile », et place les anciens andevo dans un emboîtement de relations hiérarchiques parallèles : les chrétiens par rapport à Dieu, les sujets par rapport au souverain, les andevo par rapport aux maîtres (Razafindralambo 2003 : 67).

\section{Déclinaisons de I'inégalité}

\section{Contenu des termes}

L'emploi des différents termes est rarement neutre. Il arrive que fotsy et mainty, quel que soit leur âge, s'adressent par Ingahindriana (monsieur andriana $)^{14}$ aux hommes âgés et respectés, considérés comme andriana. Ce terme est cependant couramment utilisé entre andriana, de même que la formule de salutation, un des anciens signes distinctifs de cette catégorie sociale $^{15}$. Cette formule sert actuellement de ralliement à tous ceux qui se considèrent et sont considérés comme andriana, et elle est également utilisée par certains mainty âgés. Quant au terme hova, il ne comporte aucune valeur particulière ${ }^{16}$.

Les fotsy se qualifient de fotsy, et c'est ainsi que les mainty les désignent. En revanche, les mainty n'emploient pour eux-mêmes aucune dénomination en particulier, mais sont dits mainty par les fotsy entre eux, et cela pour éviter le terme andevo jugé dépréciatif, voire insultant: ce terme contiendrait déjà en lui-même les traits de caractère supposés des andevo.

Alors que les fotsy prononcent à voix basse le mot andevo, comme de peur d'être entendu des mainty, ceux-ci, et particulièrement les jeunes (et même les jeunes enfants), l'utilisent dans les situations de confrontation, entre mainty ou entre fotsy et mainty. Dans ce contexte, andevo est compris par tous comme une insulte.

Fotsy et mainty sont compris en premier lieu dans leur sens littéral. Les fotsy ont la peau blanche, les mainty ont la peau noire. L'origine supposée

14. Ingahy est un terme honorifique adressé aux hommes dignes de respect du fait de leur âge et/ou de leur position sociale.

15. Voir page 879.

16. Il est question ici de régions anciennement andriana. Toutefois, dans certaines régions hova, où les mainty ont en face d'eux les descendants des anciens maîtres hova, les mainty parlent entre eux de tankova, qui peut être traduit par «sales hova» (RAMAMONJISOA 1984 : 43 et sq.). Une sorte d'équivalent d'Ingahindriana pour les hova pourrait être hova lahy, qualificatif qui renferme toutes les qualités supposées du hova (ibid. : 58). 
des deux groupes est explicitement liée à cette question raciale : les premiers seraient originaires d'Asie du Sud-Est, les seconds d'Afrique.

D'autres stéréotypes, d'ordre moral, sont attachés à chaque groupe. Rusés, indignes de confiance, méprisants, se considérant comme supérieurs aux mainty, de nature glissante comme leur chevelure (Ramamonjisoa 1984 : 43-44), tels sont les fotsy pour les mainty. Ces derniers sont caractérisés par une chevelure frisée ou crépue, selon les fotsy, et sont paresseux, peu instruits; ils n'ont ni le sens de la propreté, ni celui de la décence, ni celui de l'honneur; ils sont ivrognes, envieux, ont un mauvais esprit, pratiquent la sorcellerie... Le terme mainty porte, de même que son synonyme andevo, dans sa signification même la nature supposée des membres de ce groupe.

L'expression madio lamba reprend un stéréotype qui qualifie les fotsy de propres, par opposition aux mainty sales. Son emploi par les mainty a une connotation négative.

\section{Chacun sa place}

La construction de l'espace correspond à une vision du monde. La maison, dans sa forme traditionnelle, en est la concrétisation. De forme rectangulaire, son orientation est sud-nord, les ouvertures se trouvant sur la façade ouest. Trois piliers soutiennent le toit et matérialisent le centre, le nord et le sud. Aux quatre directions cardinales sont ajoutées les douze directions astrologiques qui sont ainsi spatialisées. Cependant, l'espace est essentiellement divisé en deux régions cosmiques opposées : la région nord-est et la région sud-ouest (Delivré 1974 : 148-149). La place de chaque être et de chaque objet dans la maison correspond à sa place dans l'ordre du monde. Les êtres qui occupent une position supérieure sur l'échelle sociale sont placés dans la région nord-est: les ancêtres sont invoqués au coin nord-est, où sont déposés également les objets sacrés. Les personnes importantes, ou âgées, sont placées dans la direction nord. La région sud-ouest est destinée aux sorciers ainsi qu'aux charmes maléfiques. C'est là également que s'installent les andevo. Ils ne doivent pas passer dans l'autre région, c'est-à-dire dépasser le foyer qui sépare le nord-est, avara-patana (nord du foyer), la place d'honneur, du sud-ouest, atsimom-patana (sud du foyer), la place des inférieurs et des andevo. Le lit, la vaisselle et les ustensiles de cuisine, les armes, les outils, sont placés dans la région nord-est, le mortier et le pilon, le combustible, le bétail et la volaille sont dans la région sud-ouest. Le coin sud-est, an-joro (au coin, à l'angle), est la partie la plus méprisée, la plus humble de la maison (Ottino 1986 II : 318), le coin le moins propre (Abinal \& Malzac 1993). Le pilier central tient la cohésion de l'ensemble, « il est le symbole de la solidité » (Delivré 1974 : 149).

Des valeurs symboliques sont ainsi attachées aux différentes directions cardinales et aux directions astrologiques qui leur sont liées. Le nord-est est une direction prestigieuse, la direction de la souveraineté gouvernée par 
le destin Alahamady (Ottino 1986 I : 41), destin de la souveraineté. Le nord est plus particulièrement la direction de la souveraineté guerrière, et l'est, celle de la souveraineté sacerdotale (ibid. : 273). Le souverain d'Imerina réalise les deux formes de souveraineté. Pendant les rituels royaux, le souverain s'assied toujours au nord. L'ouest est la direction du profane, des humbles; le sud est celle de la soumission, des andevo (Raison-Jourde 1991 : 87-88). La région nord-est est la région faste ; la région sud-ouest est la région néfaste (Ottino 1986 I : 244).

La direction sud n'a pas toujours eu une valeur négative. L'axe nordsud a été celui de la royauté (Belrose-Huyghues 1983: 135). Le sud est attribué au souverain, à sa famille et à ses ancêtres (ibid. : 133). La prééminence donnée au nord semble être une innovation ultérieure (ibid: 133).

$\mathrm{Ne}$ faisant pas partie des sujets, les anciens andevo n'ont pas eu leur place dans l'espace ainsi défini, ou du moins, comme c'est le cas à l'intérieur de la maison, ils sont séparés du reste de la maisonnée. D'autre part, il n'y a pas eu à proprement parler de quartiers d'andevo, bien que certains d'entre eux aient habité dans des maisons séparées, comme en témoigne cette affaire portée en 1861 devant le tribunal royal d'Antananarivo. Deux parties se disputent une rizière. Le plaignant en revendique la propriété arguant du fait que l'autre partie, qui habite la maison voisine de la sienne, est son andevo, et les biens d'un andevo appartiennent en dernier lieu à son maître. Celui qui a été accusé d'être andevo reconnaît qu'il l'a effectivement été, mais il a été racheté par sa famille. Le plaignant est débouté par la justice puisque l'autre partie est reconnue comme libre, le plaignant n'ayant pas réclamé plus tôt son droit sur le présumé andevo pourtant son voisin $^{17}$.

Les andevo ont cependant habité, pour la plupart, dans la maison même de leur maître, mais certains propriétaires ont pu regrouper leurs andevo dans des hameaux séparés.

Dans une zone de peuplement du Sud des Hautes Terres, les habitants sont divisés en deux groupes : ceux qui se disent tompon-tany (litt., Propriétaires des terres), c'est-à-dire les natifs, arrivés en premier lieu dans la région, et ceux qui sont considérés comme andevo (Evers 2001). En tant que tompon-tany, les premiers décident et accordent l'autorisation à tel ou tel nouveau venu de s'installer dans leur village. De même qu'ils décident de ranger le nouveau venu comme descendant d'ancien andevo ou non, et cela en lui attribuant soit le quartier des libres, à l'est du village, soit le quartier des supposés descendants des anciens andevo, à l'ouest, dans le but notamment d'avoir, à leur disposition, une main-d'œuvre facilement mobilisable.

Cette division des villages en quartiers se retrouve dans les villages historiques. Les descendants des anciens andriana et les descendants des

17. Arrêts royaux de justice 1861-1862, série FF Justice et passeports (FF26, Teny vita vita aminy, VAKINANKARATRA : 10R-119). 
anciens andevo occupent des quartiers séparés: le quartier nord pour les premiers, le quartier sud pour les seconds (Razafindralambo 2003: 78 et $s q$.). Dans ce contexte, la désignation de chacun selon le quartier qu'il occupe permet d'éviter l'utilisation des termes fotsy ou mainty, utilisation explicite puisque chacun garde à l'esprit que cette division en quartiers recoupe la division entre les deux groupes. Le nord est alors le quartier des fotsy, «ceux du nord », le sud celui des mainty, « ceux du sud». Ici, contrairement aux villages des nouveaux territoires qui accueillent différents groupes inconnus au préalable des uns et des autres, chacun sait ou croit savoir qui est fotsy et qui est mainty, leurs familles étant présentes depuis 1895 au moins.

Parallèlement à la division en quartiers, les morts des fotsy et ceux des mainty ne sont pas enterrés en un même lieu. Mais cela concerne plus particulièrement les descendants des anciens andriana dont l'emplacement des tombeaux se trouve à l'intérieur des fossés des villages, et ils sont généralement anciens, remontant au $\mathrm{XIX}^{\mathrm{e}}$ siècle ou même plus tôt. Ceux des descendants des anciens andevo sont très récents et en dehors des fossés. Certains descendants d'anciens andriana construisent de nouveaux tombeaux en dehors des fossés, mais à la différence des descendants d'anciens andevo, ils peuvent être rattachés à d'anciens tombeaux visibles. Avant l'abolition, les andevo ont été enterrés dans de simples caveaux disséminés en bordure des villages. La possibilité de construire des tombeaux a permis à leurs descendants de se rapprocher géographiquement des tombeaux fotsy (non andriana). Mais l'emplacement de ces tombeaux semble surtout dépendre des terrains disponibles.

Vivre ensemble mais séparément

La crainte de la sorcellerie est invoquée pour expliquer la nature des relations entre mainty et fotsy. Ils entrent rarement les uns dans les maisons des autres. Et lors des différentes circonstances qui les réunissent, ils évitent de se mettre côte à côte, que ce soit lors des assemblées villageoises, des rituels importants ou au temple protestant. Cette séparation ne se retrouve pas dans les églises catholiques ; en tout cas, elle n'y est pas aussi marquée.

Le protestantisme s'impose dès 1869 en tant que religion de la reine et donc de ses sujets. Les missionnaires catholiques se sont adressés alors aux affranchis et aux andevo, mais également aux mécontents contestant notamment l'État centralisateur personnifié par le Premier ministre hova de la reine (Kopytoff \& Miers 1993 : 295). L'assimilation du protestantisme aux hommes libres et du catholicisme aux andevo est de ce fait inexacte. En effet, bon nombre d'anciens andevo ont appartenu au protestantisme. Cette religion constitue un des éléments de l'identité, que ce soit celle des fotsy ou celle des mainty. Les ancêtres des fotsy ont souvent contribué à l'édification des temples protestants dont l'histoire est alors liée à celle de ces 
familles : «C'est le temple de nos ancêtres », déclarent-ils. De même que certains d'entre eux doivent toujours figurer parmi les dirigeants des temples parce que les terrains sur lesquels les temples ont été construits ont été offerts par leurs ancêtres. Pour les mainty, l'appartenance au temple auquel ont appartenu leurs parents, ou arrière-grands-parents, est un autre aspect de leur appartenance continue au territoire. Ce qui explique qu'ils quittent rarement leur temple ancestral pour un autre temple (Razafindralambo 2003 : 300). Et la présence massive des groupes mainty, même des membres vivant loin, lors du fetin'ny taranaka (fête des descendants), témoigne de l'importance de cette appartenance ${ }^{18}$ (ibid. : 310 et sq.) bien que celle-ci contribue à perpétuer le statut des ancêtres, anciens maîtres ou anciens andevo. D'un autre côté cependant, les ancêtres de ces derniers y trouvent une reconnaissance.

Être fidèle catholique n'a pas les mêmes implications. La conversion de certains des anciens andevo au catholicisme remonte aux débuts de l'époque coloniale. Dans ce cas, ils ont quitté le temple protestant de leurs parents (ibid. : 299). Leur adhésion ne répond à aucune revendication d'ancêtres, et ainsi n'a pas de relation avec leur statut. Cela est renforcé par la composition des fidèles des églises catholiques qui accueillent beaucoup de non-originaires établis dans les villages, y compris les affins des fotsy, contrairement aux temples protestants. Les non-originaires sont ceux qui habitent dans un village mais n'y ont pas l'attache principale qu'est le tombeau. De ce fait, ils ont un statut inférieur à celui des originaires. Mainty et fotsy ne vivent ensemble que dans l'église, car en dehors, chacun reste dans son groupe d'appartenance, originaire ou non originaire, fotsy ou mainty.

Un autre aspect des relations entre mainty et fotsy peut être observé lorsqu'ils se rencontrent. Rares sont ceux qui se saluent en ces occasions, alors qu'entre mainty ou entre fotsy on ne manque jamais de s'adresser des paroles de politesse, particulièrement lorsque l'un des interlocuteurs est un ray aman-dreny dreny (père et mère) ${ }^{19}$. On peut également remarquer qu'ils partagent rarement les mêmes repas, sauf les repas communiels liés aux rituels ancestraux.

Parents, non-parents, alliés

« Nous sommes tous parents. » Voilà comment se décrivent les villageois au chercheur étranger nouvellement arrivé. L'idéal du fihavanana $^{20}$, mode de relations fait d'unanimisme, de compréhension et de solidarité (RaisonJourde \& Randrianja 2002 : 429), s'étend ainsi au-delà du domaine de la

18. Le but de la fête est de collecter des fonds pour le temple. Tous les membres du groupe de descendance se lèvent à l'appel du nom de leur ancêtre et apportent chacun son enveloppe.

19. Personne âgée ou digne de respect.

20. Du radical havana: parent. 
parenté, la relation de parenté servant de modèle aux autres relations morales, lesquelles impliquent des droits, devoirs et obligations moraux (Bloch 1967 : 15). Ainsi, comme terme d'adresse, le terme havana est destiné aux non-parents (Bloch 1971b : 82), et comme tel, il est utilisé entre fotsy et mainty. Qualifier les fotsy de tena havana (vrai parent) implique pour les mainty une relation de « quasi-parenté malgré la barrière d'origine ancestrale, malgré les relations d'employé à employeur », avec les fotsy qui accomplissent leurs devoirs de protection (Ramamonjisoa 1984: 70). Ces devoirs de protection comprennent aides ou dons, en argent ou en vieux vêtements, offerts aux mainty. De leur côté, les fotsy désignent ainsi les mainty qui font preuve de fidélité et d'empressement à leur égard, et sur lesquels ils peuvent compter lorsqu'ils ont besoin de main-d'œuvre ou d'employés (ibid. : 69), ou pour remplacer leurs parents qui faillissent à leurs devoirs de parents (ibid. : 70). Mais ce type de relations ne lie généralement les mainty qu'aux fotsy habitant dans la capitale, et avec lesquels ils ont toujours eu des relations privilégiées, leurs ancêtres ayant été les andevo ou ayant travaillé pour ces fotsy. La limite séparant mainty et fotsy demeure : «As soon as the latter [a man of slave descent] was out of earshot, however, the man of free descent would not lose a moment to explain that the man of slave descent was not "really" (tena) his havana» (Bloch 1971b : 82). L'emploi des termes adressés généralement aux ray aman-dreny illustre cette limite. Ces termes, marque de respect et reconnaissance du statut d'aîné, ne sont pas utilisés par les fotsy vis-à-vis des mainty, et inversement. Nenisoa (maman Soa) ou Dadatsimba (père Tsimba), par exemple, deux personnes âgées mainty, sont Rasoa et Ratsimba pour les fots $y^{21}$. Dadatsima (père Tsima) ou Beberajeanne (grand-mère Rajeanne), deux personnes âgées fotsy, sont Ratsima et Rajeanne pour les mainty. L'idéal du fihavanana donne l'illusion que les relations entre voisins sont comparables aux relations entre parents, c'est-à-dire des relations entre égaux, cependant, le choix des termes employés souligne l'impossibilité d'établir des relations de parenté entre mainty et fotsy.

Une des caractéristiques que fotsy et mainty attribuent les uns aux autres est l'endogamie : «Ils se marient entre eux », disent-ils. L'endogamie a été la règle appliquée dans les anciennes catégories libres réparties en dèmes (groupes endogames et localisés). Le mariage préférentiel est entre les enfants de deux frères ou ceux d'un frère et d'une sœur (cousins parallèles patrilatéraux et cousins croisés), l'union entre enfants de deux sœurs (cousins parallèles matrilatéraux) étant strictement prohibée puisque considérés comme des germains qui n'ont pas pu être portés par le même ventre. Il s'agit d'une endogamie de statut, mais le but a été également de maintenir l'intégrité du patrimoine dont les terres constituent l'élément principal, garçons et filles héritant de manière égale. L'inscription au cadastre, dans les années 1930, a favorisé le morcellement du patrimoine au niveau des

21. Le préfixe $R a$ - est cependant un signe de respect. 
groupes de descendance et même l'individualisation de la propriété. Parallèlement, les membres des dèmes ont quitté en grand nombre leur territoire. Le mariage endogamique patrimonial n'est dès lors plus nécessaire. On peut ainsi constater que les fotsy suivent de moins en moins l'ancienne règle de l'endogamie. Ils ont plutôt une préférence pour l'exogamie, y compris ceux restés dans leur village d'origine : le choix des conjoints porte alors principalement sur des personnes originaires d'autres régions. Bien que le choix des conjoints semble ne suivre aucune règle précise, ils se marient entre fotsy.

Les andevo n'ont pas eu de parents : leur union n'a eu aucune reconnaissance officielle, le seul lien de parenté qui leur a été reconnu est celui qui unit une mère et son enfant. Ainsi, beaucoup de femmes seules sont à l'origine de groupes de descendance mainty. On ne dispose souvent que de peu d'informations concernant les conjoints de ceux qui sont nés avant 1896. À la génération suivante, nés autour des années 1900, les conjoints sont originaires d'autres villages. Cela peut être expliqué par le désir de multiplier et diversifier les alliances, et par la peur de l'inceste. Dans les générations qui suivent, la diversification des alliances en dehors des villages d'origine se poursuit. Cependant, des alliances sont dorénavant établies entre des groupes de descendance mainty de même village. Leur importance numérique est moindre par rapport aux alliances exo-villageoises. Cependant, ils sont importants pour la perpétuation des groupes de descendance puisque leurs membres qui épousent des co-villageois restent dans les villages, alors que ceux qui se marient à l'extérieur ont le choix de leur résidence, soit dans leur village, soit dans celui de leur conjoint. D'autre part, ces mariages transforment les voisins en alliés, puis en parents, et donc en partenaires pour l'entraide. Celle-ci est pratiquée dans la riziculture, mais également dans l'organisation des différents rituels. L'entraide entre parents et alliés n'est pas pratiquée dans les activités économiques non traditionnelles. Les villageois participent à une économie tournée vers l'extérieur des villages, notamment vers la capitale. Celle-ci se fournit en produits agricoles dans les villages alentour, pour lesquels elle est pourvoyeuse d'emplois. Cette culture maraîchère est limitée aux familles nucléaires, aucune entraide n'y est mise en œuvre. Des journaliers sont engagés dans certains cas, parents ou étrangers d'autres villages. La transformation des voisins en alliés, puis en parents, répond également à la crainte continuelle de la sorcellerie.

L'absence d'alliance entre fotsy et mainty constituerait la hiérarchie fondamentale (Razafindratovo 1970: 11). Dans une région du nord de l'Imerina, sur les cent quatorze mariages recensés, cinq seulement lient des fotsy et des mainty (Bloch 1971a : 169). Les unions entre jeunes garçons mainty et jeunes filles fotsy sont diversement appréciées dans l'ancienne cité royale d'Ambohimanga, «honni des uns, applaudi des autres » (Razafintsalama 1973 : 110), l'idéologie ancienne interdisant particulièrement l'alliance entre andriana et mainty, est encore en vigueur, et les unions entre andriana et hova restent fortement déconseillées (ibid. : 199). Les unions mainty-fotsy 
existent donc mais elles sont rares. Aucune interdiction cependant n'empêche les relations sexuelles entre personnes des deux groupes, « un privilège de supérieurs » pour jeunes hommes fotsy (Ramamonjisoa 1984 : 53). Les enfants issus de ces unions non légitimes ne sont reconnus ni par le père ni par la famille de celui-ci, même si la filiation est connue de tous grâce aux rumeurs.

Les unions légitimes entre mainty et fotsy peuvent concerner des personnes originaires du même village ou de la même région. Les familles des deux conjoints savent alors à quelle catégorie chacun appartient. Ou croient savoir. En effet, il arrive que des familles établies depuis longtemps dans un village, ou dans la région, sont considérées comme des originaires. Suivant les stéréotypes identifiant les différentes catégories, ils sont rangés soit parmi les fotsy soit parmi les mainty : fotsy s'ils possèdent une maison et des terres, s'ils ont de l'instruction ; mainty lorsqu'ils ont quitté leur village d'origine au lendemain de l'abolition, et ne possèdent pas de terres là où ils ont choisi de vivre, ou l'ont acquis récemment, et leurs parents ont été au service des familles fotsy. Ils ont établi des alliances avec des groupes originaires, avec les groupes fotsy s'ils sont considérés comme fotsy, avec les groupes mainty s'ils sont considérés comme mainty. Ou inversement, ils sont considérés comme fotsy parce qu'ils ont pu établir des alliances avec des groupes fotsy (et particulièrement, comme andriana s'ils sont mariés à des andriana), ou mainty parce qu'ils ont établi des alliances avec des groupes mainty. Les conjoints connus comme non originaires sont mainty ou fotsy, selon les mêmes représentations appliquées aux groupes originaires.

La famille des conjoints fotsy tait généralement l'existence de ces unions avec des mainty. Comme ce père dont deux des filles ont épousé un mainty, qui ne mentionne que ces belles-filles et gendres qu'il juge conformes à sa position de fotsy, en l'occurrence l'une est la fille d'un boucher, d'après lui originaire du même village (en réalité, un non-originaire qui a pu acquérir beaucoup de terres dans la région).

La conclusion de ces unions mainty-fotsy se fait rarement sans difficulté. Une jeune fille a décidé d'épouser un mainty de la région, mais du village voisin. Les villageois se souviennent à quel point cet événement a été un véritable drame pour les familles des deux jeunes gens, toutes deux également opposées au mariage. Le couple a acheté un terrain dans le village d'origine de l'épouse et continue d'y vivre. Les frères et sœurs de l'épouse vivent maintenant en dehors de la région, aussi le couple n'a pas de relations proches avec eux. Quelques autres membres de la famille sont restés au village, mais leur position dans la parenté est suffisamment éloignée pour qu'ils aient des relations avec le couple. Les relations du couple avec la famille mainty de l'époux sont presque aussi distantes. D'après les villageois, les relations sont inexistantes, le couple ne vient jamais rendre visite aux parents âgés de l'époux. Ce qui est inexact d'après les membres de la famille, et il arrive que ce sont les parents qui se déplacent chez eux. La 
rupture la plus importante cependant s'est effectuée lorsque le couple, auparavant protestant, s'est converti à un autre mouvement religieux. Cette conversion équivaut à se mettre en retrait du groupe de descendance puisque l'appartenance au temple protestant constitue un élément de l'identité aussi bien des groupes fotsy que mainty. D'autre part, être membre de ce mouvement religieux permet de ne pas participer aux cérémonies qui rassemblent le groupe de descendance, en particulier aux rituels liés aux ancêtres. Les enfants mariés du couple suivent un autre chemin. Leur fille cadette a épousé un non-originaire considéré comme mainty. Le mari de la fille aînée, en revanche, est originaire du même village que le père. Il est mainty et protestant. Le couple habite à proximité des parents de l'épouse. La raison invoquée est d'ordre matériel: les parents y ont une maison disponible. Néanmoins, l'épouse appartient dorénavant au temple protestant de son mari, qui est également celui de la famille de son père. Elle a choisi ainsi d'appartenir au groupe mainty de son père, choix renforcé par son mariage avec un mainty du même village que ce dernier. La conversion des parents consacre la rupture avec le groupe fotsy de la mère.

Une histoire similaire s'est déroulée entre une jeune femme fotsy et un jeune homme non originaire considéré comme mainty. Comme précédemment, le projet des jeunes gens a provoqué un véritable drame. Le jeune homme est arrivé avec sa famille dans la région dans les années 1960. Plusieurs raisons ont contribué à ce qu'il soit déclaré mainty. Tout d'abord, le village d'où sa famille est originaire est un village d'anciens andevo. $\mathrm{Ou}$ du moins, il aurait déclaré que les habitants de ce village font partie de ses parents, et suivant les représentations, on est mainty quand on a des parents mainty. En d'autres occasions, il n'aurait rien répondu à une question relative à sa catégorie sociale. Ce silence est interprété comme un aveu, puisque dit-on, s'il avait été fotsy, il n'aurait pas hésité à répondre. La mère de la jeune femme a fini par consentir au mariage après être allée consulter un mpitaiza $a^{22}$, estimant que l'attachement de sa fille est «anormal ». Le mpitaiza aurait déclaré que la jeune femme a été ensorcelée par le fiancé, et que la séparation allait entraîner leur mort à tous les deux. Par peur de la sorcellerie, la famille de la jeune femme n'a pas assisté au repas de mariage préparé par la famille du fiancé. Le couple s'est établi dans une maison héritée par l'épouse. Celle-ci a déclaré qu'elle sera enterrée avec ses parents ; ses enfants vont probablement la suivre. Son mari est maintenant plus ou moins bien accepté par la famille de son épouse, bien qu'il reste pour eux un mainty. Les enfants, en revanche, sont considérés comme fotsy.

L'épouse mainty n'a aucune possibilité d'intégrer le tombeau de son conjoint fotsy. Cependant, quelques exceptions sont à signaler : des épouses mainty ont été enterrées dans le tombeau de leurs époux andriana, bien que

22. Le mpitaiza (litt., celui qui prend soin) joue la fonction de devin-guérisseur et d'intercesseur auprès des ancêtres royaux. La sorcellerie figure parmi les principaux motifs de consultation. 
leurs voisins de la région affirment le contraire. Les enfants issus de ces mariages sont tous enterrés dans le tombeau de leur père andriana, y compris la fille mariée, dont le mari, originaire de la région, est hova. À la génération suivante, tous les partenaires de mariage sont originaires d'autres régions, mais toujours fotsy. Le choix des ancêtres appelés lors du fetin'ny taranaka ${ }^{23}$ montre que les membres actuels de ce groupe de descendance ont choisi l'identité andriana de leur grand-père : ils font désormais référence aux enfants de celui-ci, écartant ainsi leur grand-mère mainty. D'autre part, ils désignent comme membres de leur famille ceux qui ont choisi également l'identité andriana, bien que le lien de parenté qui les lie soit par leurs grands-mères mainty qui sont sœurs. Ils n'ont aucune relation avec leurs parents mainty. Ces unions entre mainty et andriana ont pu aboutir, dit-on également dans ces cas, par l'utilisation par les mainty de la sorcellerie.

Pour chaque individu demeure la possibilité de choix entre les tombeaux de ses quatre grands-parents de qui il tient son statut, ses droits, principalement sur le patrimoine du groupe, et ses obligations et devoirs ${ }^{24}$. Dans les mariages endogamiques entre parents, les conjoints appartiennent au même groupe de descendance, et donc au même tombeau. Il en va de même de leurs enfants. Pour les conjoints des unions entre personnes de catégories différentes, et les enfants issus de ces unions, le droit de l'épouse au tombeau de son époux peut être limité. Il est habituel qu'une épouse soit enterrée dans le tombeau de son époux, ainsi que leurs enfants. Cependant, certains tombeaux anciens sont interdits aux personnes n'appartenant pas au même groupe de descendance, ou aux personnes de catégories ou groupes différents. Par exemple, des tombeaux andriana sont interdits aux nonandriana ou aux andriana de rang inférieur. Dans ce cas, l'épouse peut se voir refuser l'entrée du tombeau de son mari. L'exclusion des tombeaux est généralement tue par les descendants, exclusion de l'épouse hova du tombeau de son mari andriana, exclusion de l'épouse mainty de celui de son mari fotsy.

Il arrive également que l'épouse d'un rang supérieur à celui de son époux choisisse de ne pas être enterrée avec lui. Celle-ci rejoint alors le tombeau de sa famille, généralement celui de son père. Les enfants peuvent toutefois opter soit pour le tombeau de leur père soit pour celui de leur mère, sous réserve cependant du consentement du groupe attaché au tombeau. Il en est ainsi lorsque les descendants de mainty et fotsy optent pour l'identité fotsy. Cela leur permet par la suite de réclamer et d'exercer leurs droits sur le patrimoine du groupe fotsy, et notamment pour les andriana, des terres qu'ils sont les seuls à posséder sur et autour des collines historiques.

23. Voir note 18.

24. Le recrutement de ces groupes de descendance est cognatique : chaque membre peut appartenir au groupe de chacun de ses quatre grands-parents (grands-pères maternel et paternel, grands-mères maternelle et paternelle). 
Le choix de l'identité fotsy ou mainty a d'autres implications, notamment au niveau de la communauté villageoise. Être fotsy, et particulièrement andriana, a toujours du prestige. Des hova de la région déclarent qu'ils sont originaires de l'ancienne colline des andriana, ce qui signifierait qu'ils seraient andriana mais non pas hova, ou mettent en avant leur lien de parenté avec les andriana. D'autre part, les différentes positions de responsabilité, électives ou désignées, aussi bien au sein des temples que dans l'administration des villages, sont occupées par des fotsy, et leur sont, d'une certaine manière, réservées. Les responsabilités détenues par les mainty sont celles que les fotsy leur ont concédées. Les personnes issues de groupes désignés comme fotsy et andriana même si un de leurs ancêtres est connu de tous comme mainty, ont le même statut que les autres fotsy, et on s'adresse à eux par les salutations réservées aux andriana ${ }^{25}$.

L'identité de l'individu ne dépend pas du lieu de son ensevelissement (Ottino 1998 : 39), d'autant plus que ce lieu peut être provisoire. Il peut, en revanche, contribuer à déterminer celle de ses descendants (ibid.) : une femme mainty enterrée avec son mari andriana reste une femme mainty. Leurs enfants peuvent bénéficier et renforcer l'identité liée au tombeau de leur père. La résidence détermine les droits effectivement exercés, notamment sur les terres à cultiver, et les différentes obligations, par exemple dans les rituels (Vogel 1973, 1982). Tombeau, patrimoine et statut sont donc liés à l'appartenance à un groupe de descendance, et inversement.

La barrière sociale séparant les mainty et les fotsy est inscrite dans l'espace et se concrétise dans les relations quotidiennes entre les deux groupes. La place de chaque groupe peut être expliquée par les valeurs attachées aux directions cardinales et astrologiques, qui confirme également son statut. Les comportements des uns vis-à-vis des autres confirment la séparation géographique et leurs relations sont caractérisées par l'évitement expliqué par la peur de la sorcellerie. On pourrait penser qu'une impureté est attachée aux descendants des anciens andevo et explique cette barrière. Le simple contact physique peut polluer et nécessite un rituel de purification (Evers 1995 : 176). Cependant, parmi les tâches des anciens andevo figure la préparation des repas. Les femmes ont également été les nourrices des enfants des maîtres. D'autre part, les relations sexuelles entre les libres et nonlibres, puis entre mainty et fotsy, ne sont pas prohibées. Le problème réside dans le mariage en tant que lien social.

La séparation est manifeste au niveau verbal. Les termes de parenté séparent les mainty et les fotsy, et excluent ainsi ceux qui ne peuvent devenir des parents, ni des alliés, c'est-à-dire les mainty pour les fotsy, et inversement, et cela même au niveau d'une parenté «fictive». Certains anciens

25. Les hova et les andriana auraient une attitude différente. Ces derniers toléreraient les unions avec les mainty, contrairement aux hova qui les refuseraient de manière plus catégorique. Ce que semble confirmer la situation dans le village hova d'Ilafy (RAMAMONIISOA 1984 ; RAZAFINDRATOVO 1970). 
andevo ont été enterrés dans le tombeau de leurs anciens maîtres. La place qui leur est accordée à l'intérieur du tombeau, près de l'ouverture à l'ouest, dit-on, reflète cependant leur position : on ne peut leur accorder le statut d'un membre de la famille; ils ne peuvent faire partie de la famille du maître ${ }^{26}$. On est donc fotsy ou mainty, chaque identité s'excluant l'une l'autre. C'est ce que montre le choix que doit effectuer, quand cela est possible, les enfants issus des unions entre mainty et fotsy.

\section{Ancêtres et résorption de l'inégalité ?}

Ny andevo tsy mba raza: l'andevo n'a pas d'ancêtre et ne peut le devenir. Il n'a pas eu le droit d'établir des liens de parenté. Sa famille a été limitée à la mère et à ses enfants. À sa mort, « on le porte quelque part en terre dans les biens de son propriétaire et c'est tout » (André 1899 : 96). Le libre, en revanche, s'inscrit dans un enchevêtrement de liens, dont l'ancêtre et le tombeau sont le point de départ et l'aboutissement, mais en sont également la cristallisation. La transformation du mort en ancêtre qui dispense la bénédiction à ses descendants commence par le rituel d'enterrement.

Le rituel d'enterrement n'a pas été accompli pour les anciens andevo. On dit de leurs morts qu'ils ont été jetés dans les caveaux sans cérémonie. Cette absence de rituel est liée au fait qu'ils ne peuvent devenir ancêtres (Evers 2001 : 125 et sq.).

Le tombeau est une construction solide, visible, en pierres. Le caveau représente l'image inverse. Il est surmonté d'un petit monticule de terre, à peine visible. Les corps y sont placés sans ordonnancement (ordre dans la parenté, selon le sexe, ou l'âge...), et par terre. Le caveau évoque la promiscuité, contrairement au tombeau dans lequel les morts reposent sur des lits en pierre. D'autre part, les caveaux ne sont pas disposés sur un emplacement précis, ils sont généralement en dehors et en bordure des villages, ou près des terres de culture. Les tombeaux des andriana se trouvent eux à l'intérieur des anciens villages situés sur les collines. Ceux des autres fotsy sont disséminés autour des villages. Il est cependant fréquent que les tombeaux soient rassemblés en un seul lieu. Les tombeaux des fotsy sont généralement anciens. Les tombeaux des mainty sont tous récents. Ils ont été construits après l'abolition. Même leur emplacement a changé puisqu'ils sont situés avec les autres tombeaux.

La construction des tombeaux permet dorénavant l'accomplissement du rituel d'ancestralisation, le rituel du famadihana (litt., retournement). Il consiste à sortir le mort du tombeau, à le réenvelopper dans de nouveaux linceuls, puis à le placer dans son tombeau définitif. Le mort peut être replacé dans le tombeau d'où on vient de le sortir. Ou lorsque le mort a

26. Avant l'abolition, l'adoption de l'andevo par le maître après son affranchissement peut le consacrer comme membre à part entière de la famille de celui-ci. 
été enterré dans une tombe provisoire ${ }^{27}$ ou dans une autre région, il est ramené dans son tombeau ancestral: c'est le «return famadihana» ou «famadihana de transfert» (Bloch 1971c: 146). Un troisième type de rituel consiste à déplacer le corps dans un tombeau qui vient d'être construit lors d'un famadihana d'inauguration. Dans certains cas plus rares, le mort est déplacé d'un tombeau à un autre. Les familles peuvent également profiter de l'ouverture de leur tombeau, comme lors d'un enterrement, pour réenvelopper leurs morts de nouveaux linceuls sans les sortir du tombeau. Ce sont surtout les fotsy qui procèdent ainsi. D'autant plus que certains groupes andriana ne pratiquent pas le famadihana. Cependant, ils effectuent le famadihana de transfert et d'inauguration et ne qualifient de « vrai famadihana» que le rituel au cours duquel les morts ne sont que réenveloppés de nouveaux linceuls.

Le famadihana ne peut être accompli qu'une seule fois pour chaque défunt, quelques années après l'enterrement, lorsque le corps est $\sec ^{28}$. Ce rituel consacre le statut d'ancêtre dispensateur de bénédiction. La bénédiction assure les bienfaits attendus de la vie : la santé, la richesse, mais également la perpétuation du groupe par la reproduction de ses membres (la fertilité) et par l'unité des descendants d'un même ancêtre, la bénédiction se transmettant d'une génération à une autre. Le tombeau est ainsi le point de départ de la constitution d'un groupe de descendance qui ne peut exister sans lui.

La question des ancêtres met en relief deux statuts différents au sein des communautés villageoises : les tompon-tany, c'est-à-dire les originaires, et les non-originaires. Les originaires peuvent justifier de la présence d'ancêtres dans le village (ou la région), ce qui n'est pas le cas des non-originaires. Cette différence de statut est manifeste dans toutes les circonstances qui réunissent les villageois, essentiellement les assemblées villageoises et religieuses, et les rituels du famadihana. Les non-originaires n'y assistent pas. Et ceux qui viennent aux assemblées s'abstiennent de prendre la parole. Les non-originaires mariés à des originaires se conduisent et sont considérés, dans ce contexte, comme faisant partie du groupe de descendance de leur conjoint.

Aux mainty, désormais possesseurs de tombeaux et d'ancêtres enterrés au village, la qualité d'originaires n'est pas discutée. À cet égard, l'accomplissement du rituel du famadihana est primordial. Ceux qui en ont les moyens l'organisent le plus fréquemment possible. La présence des originaires fotsy à ces fêtes constitue une reconnaissance des ancêtres des mainty et du statut d'originaires de leurs descendants. Une des principales différences entre mainty et fotsy repose cependant sur l'ancienneté de leurs ancêtres et de

27. Comme lorsqu'un enterrement a eu lieu dans le même tombeau quelque temps plus tôt, et le délai légal de fermeture du tombeau n'est pas encore atteint. Le mort est alors enterré à proximité du tombeau, quelquefois dans un cercueil.

28. Humide, il a un caractère polluant. 
leurs groupes de descendance. Pour les mainty, les ancêtres les plus lointains dont ils se réclament remontent tous à l'abolition. Le lien des descendants avec les ancêtres, et par là, avec le territoire, est réactualisé et réaffirmé régulièrement. Pour les fotsy, en revanche, et particulièrement les andriana, les débuts de leur groupe se confondent avec l'histoire la plus ancienne des villages. Cette présence ancienne et continue de leurs ancêtres suffit à marquer leur appartenance au territoire.

Le rituel du famadihana est important à plusieurs titres. Il détermine l'emplacement définitif des morts et permet de les instituer en ancêtres. Il n'est possible que lorsque le groupe a construit un tombeau dont la solidité et le caractère visible symbolisent le groupe de descendance. La survie du groupe est assurée par la bénédiction des ancêtres. Les ancêtres présentent cependant deux aspects contradictoires. D'un côté, les anciens andevo n'ont pas eu d'ancêtres. La construction de tombeau et l'accomplissement du rituel du famadihana permettent à leurs descendants de construire des ancêtres et ainsi d'acquérir un nouveau statut: celui d'originaires. D'un autre côté, cependant, les ancêtres dont les mainty se réclament restent d'anciens andevo. De ce fait, leur ancien statut se perpétue, sans que leurs descendants aient la possibilité de l'effacer.

L'ancien système hiérarchique des Hautes Terres a divisé la population en deux groupes : les libres et les non-libres. Le souverain se trouve au sommet, et c'est par rapport à lui qu'est déterminée la place de chacun au sein de ce cadre hiérarchique. Les termes qui ont organisé cette hiérarchie continuent d'être utilisés, bien que ce système n'ait plus cours puisque la Constitution de la République de Madagascar institue citoyens tous les Malgaches quels qu'aient été leurs ancêtres. Le contenu de ces termes a cependant changé. L'andriana n'est plus le membre d'un groupe endogame dont une préséance rituelle et quelques privilèges manifestent la supériorité. À l'autre extrémité de l'échelle sociale, l'andevo n'est plus celui qui appartient au fanjakana de son maître, qui est dans l'incapacité d'établir des liens de parenté et qui n'a pas d'ancêtres. Les anciens statuts sont ainsi reproduits. Mais à ce maintien des anciens termes se superpose désormais une division en deux catégories sociales: les fotsy et les mainty.

Des représentations morales et physiques ont été élaborées par l'une et l'autre catégorie. Elles opposent les mainty, supposés d'origine africaine donc arrivés en dernier lieu, aux fotsy, qui seraient venus d'Asie du Sud-Est. Identifier les anciens andevo à des étrangers non malgaches permet de nier que des anciens sujets libres ont pu devenir andevo. Ces représentations, définissant chacune des deux catégories, sont opposées terme à terme : AfriqueAsie, sale-propre, noir-blanc, paresseux-travailleur, ivresse-sobriété... et sud-nord ou ouest-est. Bien que fotsy et mainty partagent le même espace 
villageois, ils sont séparés en quartiers. La répartition des habitations peut être expliquée par les valeurs attachées aux points cardinaux. D'autre part, ce regroupement en quartiers permet aux uns et aux autres de constituer un voisinage de parents et/ou d'alliés. Leurs relations sont caractérisées par l'évitement, dont la ligne passe par la division en quartiers. Ces conduites d'évitement sont motivées par la crainte réciproque de la sorcellerie contre laquelle une des principales protections est de vivre avec les parents et/ou les alliés. Les règles de mariage pratiquées par les groupes mainty visent à transformer les voisins en alliés, puis en parents : choix des partenaires en dehors du village au lendemain de l'abolition, et, dans chacune des générations suivantes, quelques partenaires du même village afin d'établir des alliances avec les autres groupes mainty du village. Une des règles qui régissent le mariage est donc la préférence pour les partenaires de mêmes catégories sociales : entre fotsy et entre mainty. Les mariages entre mainty et fotsy ne sont pas formellement interdits, mais le fait que ces mariages sont généralement passés sous silence par les familles concernées ainsi que les rumeurs qui circulent sur le sujet montrent qu'ils ne font pas partie des normes acceptées par la société. D'autant plus que les enfants issus de ces unions doivent choisir entre le groupe fotsy ou le groupe mainty de leur père ou de leur mère. Ou plus exactement, le groupe fotsy décide si les enfants peuvent faire partie de leur groupe ou non. L'appartenance à un groupe de descendance fotsy est établie par la filiation avec l'ancêtre du groupe. Ceux qui appartiennent au groupe mainty suivent les règles de mariage mainty, c'est-à-dire partenaire mainty, et répétition d'alliance avec les autres groupes mainty du même village. Ceux qui appartiennent aux groupes fotsy épousent des fotsy non originaires. Mainty et fotsy ne peuvent donc être parents, et chaque identité s'exclut l'une l'autre: on ne peut être mainty et fotsy en même temps alors qu'un individu né de père et mère andriana et hova peut être andriana ou hova selon les circonstances.

Un des traits qui définissent les andevo est leur incapacité à avoir d'ancêtres du fait de leur état de non-libre. La qualité d'ancêtre est intimement liée à l'existence de tombeau. Dans le tombeau, les morts sont placés selon leur place dans le groupe de parenté, et selon un ordre déterminé par le sexe et l'âge. Le tombeau réunit donc ceux qui se reconnaissent et qui sont reconnus comme parents. Les andevo ont auparavant été enterrés dans de simples caveaux dans lesquels les corps des morts ont été jetés par terre, sans aucun ordre. Leur déplacement dans des tombeaux nouvellement construits permet l'accomplissement de rituels qui transforment les morts en ancêtres, de l'enterrement au rituel du famadihana qui consacre le statut d'ancêtre. Le mort est désormais sec et donc dispensateur de bénédiction : la bénédiction court le long du groupe de descendance ; elle est le garant de son existence et de sa survie. Les descendants des anciens andevo sont désormais membres de groupes de descendance. Contrairement aux ancêtres des fotsy, cependant, leurs ancêtres remontent à l'abolition de 1896, alors que les fotsy se réclament d'ancêtres liés à l'histoire la plus ancienne des 
villages. L'abolition, comme l'affranchissement, a donné la capacité aux anciens andevo de créer les relations sociales, dont les relations de parenté. Leur intégration dans la société définie par les fotsy se fait par l'adoption des valeurs fotsy. Les mainty sont maintenant des originaires des villages dans lesquels leurs ancêtres sont enterrés. Mais ils n'ont pas acquis les mêmes droits que leurs co-originaires fotsy, ceux qu'ils exercent sont ceux que ces derniers leur ont accordés.

La division mainty-fotsy constitue une grille de lecture à travers laquelle les relations entre villageois peuvent être comprises. Elle commande leurs comportements, les répartit en différentes classes (ceux qu'on peut épouser, à qui on doit faire appel pour exécuter certaines tâches...). Cependant, mainty et fotsy correspondent à une construction. Les mainty n'appartiennent pas à une catégorie définie historiquement et donc par des règles et une tradition culturelle et historique (Razafintsalama 1973 : 204). Ces représentations des mainty élaborées par les fotsy correspondent à une inversion de leur image, et ont pour fonction de maintenir l'inégalité entre mainty et fotsy en l'absence des lois qui ont tenu séparés les libres des non-libres. Les mainty restent ainsi dans une position d'éternels dépendants, les fotsy dans leur statut d'anciens dominants.

Université d'Antananarivo, Madagascar.

\section{BIBLIOGRAPHIE}

Abinal, F. G. P. \& Malzac, V.

1993 [1888] Dictionnaire malgache-fançais, Antananarivo, Fianarantsoa.

ANDRÉ, C.

1899 De l'esclavage à Madagascar, Paris, Arthur Rousseau.

Belrose-Huyghues, V.

1983 «Structure et symbolique de l'espace royal en Imerina», in F. RAISONJourde (dir.), Les souverains de Madagascar, Paris, Karthala : 125-151.

BLOCH, M.

1967 «L'extension de la notion de "havana" dans la société merina rurale », Bulletin de l'Académie malgache, XLIII (2) : 15-16.

1971a «The Implications of Marriage Rules and Descent: Categories for Merina Social Structures », American Anthropologist, 73 (1) : 164-178.

1971b « The Moral and Tactical Meaning of Kinship Terms », Man, 6 (1) : 79-87.

1971c Placing the Dead. Tombs, Ancestral Villages, and Kinship Organization in Madagascar, London-New York, Seminar Press.

1980 «Modes of Production and Slavery in Madagascar: Two Case Studies », in J. L. Watson (ed.), Asian and African Systems of Slavery, Oxford, Basil Blackwell : 100-134. 
1994 «The Slaves, the King, and Mary in the Slums of Antananarivo », in N. THOMAS \& C. Humphrey (eds.), Shamanism, History and State, Ann Arbor, University of Michigan Press : 133-145.

1999 «Commensality and Poisining», Social Research, 66 (1) : 133-149.

Botte, R. (dir.)

2000 «De l'esclavage et du daltonisme dans les sciences sociales », Journal des Africanistes, 70 (1-2) : 7-42.

Condominas, G. (dir.)

1998 Formes extrêmes de dépendance. Contributions à l'étude de l'esclavage en Asie du Sud-Est, Paris, Éditions de l'EHESS.

Delivré, A.

1974 L'histoire des rois d'Imerina, Paris, Klinckseck.

Domenichini-Ramiaramanana, B. \& Domenichini, J.-P.

1980 «Regards croisés sur les grands sycomores, ou l'armée noire des anciens princes d'Imerina », ASEMI, Cheminements, 11 (1-4) : 55-95.

1982 «Aspects de l'esclavage sous la monarchie merina d'après les textes législatifs et réglementaires », Omaly sy Anio, $15: 53-98$.

1997 « 1877 : une abolition de l'esclavage ? », in I. RAкото (dir.), op. cit. : 233-244.

1998 «L"'esclavage" dans la société malgache », in G. Condominas (dir.), Formes extrêmes de dépendance. Contributions à l'étude de l'esclavage en Asie du Sud-Est, Paris, Éditions de l'EHESS.

Evers, S.

1995 « Stigmatization as a Self-Perpetuating Process », in S. Evers \& M. SPINDLER (eds.), Cultures of Madagascar. Ebb and Flow of Influences, Working Paper Series, 2, Leiden, International Institute for Asian Studies : 157-185.

1999 «The Construction of History and Culture in the Southern Highlands : Tombs, Slaves and Ancestors », in K. Middleton (ed.), Ancestor, Power and History, Leiden, Brill : 257-282.

2001 Constructing History, Culture and Inequality. The Betsileo in the Extreme Southern Highlands of Madagascar, thèse de doctorat, Amsterdam, Université d'Amsterdam.

Kopytoff, I. \& Miers, S. (eds.)

1977 Slavery in Africa. Historical and Anthropological Perspectives, Madison, University of Wisconsin Press.

1993 Madagascar et le christianisme, Paris, Karthala (« Histoire Ecuménique ») ; ACCT, Antananarivo, Ambozontany.

Meillassoux, C.

1986 Anthropologie de l'esclavage. Le ventre de fer et d'argent, Pratiques théoriques, Paris, PUF.

Meillassoux, C. (dir.)

1975 L'esclavage en Afrique précoloniale, Paris, François Maspero («Bibliothèque d'Anthropologie »). 
OtTINo, P.

1986 L'Étrangère intime. Essai d'anthropologie de la civilisation de l'ancien Madagascar, 2 tomes, Paris, Éditions des archives contemporaines.

1998 Les champs de l'ancestralité à Madagascar. Parenté, alliance et patrimoine, Paris, Karthala-ORSTOM.

PAtTerson, O.

1982 Slavery and Social Death. A Comparative Study, Cambridge-London, Harvard University Press.

RAFIDINARIVO RAKOTOLAHY, C.

2000 «Le référent de l'esclavage dans les représentations transactionnelles marchandes à Madagascar », Journal des Africanistes, 70 (1-2) : 123-144.

RAISON, J.-P.

1977 «Perception et réalisation de l'espace dans la société merina », Annales ESC, $3: 412-432$.

RAISON-JOURDE, F.

1991 Bible et pouvoir à Madagascar au XIX ${ }^{e}$ siècle. Invention d'une identité chrétienne et construction de l'État (1780-1880), Paris, Karthala.

RAison-Jourde, F. \& RANDRIANJa, S. (dir.)

2002 La nation malgache au défi de l'ethnicité, Paris, Karthala.

RAKото, I. (dir.)

1997 L'esclavage à Madagascar. Aspects historiques et résurgences contemporaines, Actes du colloque international sur l'Esclavage, 24-28 septembre 1996, Antananarivo, Institut de Civilisations, Musée d'Art et d'Archéologie.

Rakotomalala, M. \& Razafimbelo, C.

1985 «Le problème d'intégration sociale chez les Makoa de l'Antsihanaka», Omaly sy Anio, 21-22: 93-113.

RAMAMONJISOA, J.

1984 «"Blancs et Noirs". Les dimensions de l'inégalité sociale», Cahiers des Sciences sociales: $39-75$.

RAmanantsoa Ramarcel, B.

1997 « Mainty=Andevo, un amalgame statutaire de l'Imerina », in I. RAкото (dir.), op. cit. : $147-160$.

RANDRIAMARO, J. R.

1997a PADESM et luttes politiques à Madagascar. De la fin de la Deuxième Guerre mondiale à la naissance du PSD, Paris, Karthala.

$1997 \mathrm{~b}$ «L'émergence politique des mainty et andevo au Xx ${ }^{\mathrm{e}}$ siècle », in I. RАкото (dir.), op. cit. : 357-381.

RANTOANDRO, G.

1997 « Après l'abolition de l'esclavage à Madagascar. Le devenir immédiat des esclaves émancipés », in I. RAKOTO (dir.), op. cit. : 273-289. 
RAZAFINDRALAMBO, L. N.

2003 La notion d'esclave en Imerina (Madagascar) : ancienne servitude et aspects actuels de la dépendance, Thèse de doctorat, Nanterre, Université Paris 10.

RAZAFINDRATOVO, J.

1968a «Étude du village d'Ilafy » ( $1^{\mathrm{re}}$ partie), Annales de l'Université de Madagascar («Lettres et Sciences humaines ») 8:3-15.

1968b «Étude du village d'Ilafy » (2e partie), Annales de l'Université de Madagascar («Lettres et Sciences humaines ») 9: 47-71.

1969 «Étude du village d’Ilafy » (3 $3^{\mathrm{e}}$ partie), Annales de l'Université de Madagascar («Lettres et Sciences humaines ») $10: 51-74$.

1970 Hiérarchie et alliance dans un village de l'Imerina, Thèse de doctorat, 2 vol., EPHE, Paris.

RaZafintsalama, A.

1973 Les Tsimahafotsy d'Ambohimanga, Faculté des Lettres, Université de Madagascar, Cahiers du Centre de sociologie et d'anthropologie sociale, 1.

Vogel, C.

1973 «Stratégies d'équilibre et d'expansion dans une population régionale d'Imerina », ASEMI, IV (4) : 35-51.

1982 Les quatre-mères d'Ambohibaho. Étude d'une population régionale d'Imerina (Madagascar), Paris, Selaf («Langues et civilisations de l'Asie du Sud-Est et du Monde insulindien »).

WATSON, J. L. (ed.)

1980 Asian and African Systems of Slavery, Oxford, Basil Blackwell.

WATSON, J. L.

1980 «Slavery as an Institution: Open and Closed Systems », in J. L. WATSON (ed.), op. cit. : 1-15.

RÉSUMÉ

Ce texte est une réflexion sur les relations sociales au sein de la société d'Imerina (Hautes Terres malgaches). Celles-ci ne peuvent être comprises que dans le cadre de la division de la population en deux catégories sociales principales, dont l'appartenance est déterminée par le statut supposé des ancêtres. Les représentations, et les comportements qu'elles régissent, marquent la séparation entre membres de catégories différentes dans la vie quotidienne, et contribuent ainsi à maintenir la hiérarchie sociale. Le processus par lequel les relations entre catégories sociales différentes se rigidifient est donc analysé ici. On peut y voir également un aspect paradoxal du changement : il peut contribuer à renforcer les anciennes positions. 


\section{ABSTRACT}

Inequality, Exclusion, Mental Representations on the Central Highlands of Madagascar. - Social relations among the Merina on the highlands of Madagascar can be understood only in relation to the division of the population into two major social categories based on the assumed status of ancestors. The resulting conceptions and behaviors mark a separation between members of these two categories in everyday life and thus help maintain the social hierarchy. The process whereby the relations between different social categories become rigid is analyzed. It represents a paradoxical aspect of change, which can reinforce former positions.

Mots-clés/Keywords: Hautes Terres de Madagascar, ancêtres, construction, esclavage, exclusion, groupe de descendance, identité, inégalité, stéréotypes/Highlands of Madagascar, ancestors, slavery, exclusion, descent group, identity stereotypes, inequality. 